\title{
Decreased PSD95 expression in medial prefrontal cortex (mPFC) was associated with cognitive impairment induced by sevoflurane anesthesia ${ }^{*}$
}

\author{
Yun-zhi LING ${ }^{\S 1,2}$, Wei MA ${ }^{\S 3}$, Li YU ${ }^{4}$, Ye ZHANG ${ }^{\dagger 1}$, Qi-sheng LIANG ${ }^{2}$ \\ ( ${ }^{1}$ Department of Anesthesiology, the Second Affiliated Hospital of Anhui Medical University, Anhui 230601, China) \\ ( ${ }^{2}$ Department of Anesthesiology, the First Affiliated Hospital of Bengbu Medical College, Anhui 233004, China) \\ $\left({ }^{3}\right.$ Department of Preventive Treatment of Disease, Yulin City Hospital of Traditional Chinese Medicine, Shanxi 719000, China) \\ $\left({ }^{4}\right.$ Department of Laboratory Medicine, Bengbu Medical College, Anhui 233003, China) \\ †E-mail: zhang ye011@163.com \\ Received Jan. 5, 2015; Revision accepted Apr. 29, 2015; Crosschecked Aug. 10, 2015
}

\begin{abstract}
Objective: Though sevoflurane has been widely used as an anesthetic in surgery, recent studies have shown that exposure to sevoflurane alone could lead to postoperative cognitive dysfunction (POCD), of which the mechanisms still remain largely unknown. The medial prefrontal cortex (mPFC) is known to be implicated in various cognitive impairments, including working memory and attentional processes. In the present study, we tried to identify dysregulated gene expression in mPFC and investigate the underlying mechanisms involved in POCD. Methods: Behavioral tests, including elevated plus-maze, O-maze, and Y-maze tests, were performed on Wistar rats exposed to sevoflurane. Whole-genome mRNA profiling of mPFC from Wistar rats after exposure to sevoflurane was carried out. Real-time polymerase chain reaction (PCR) was done to verify the differentially expressed genes. Results: Significant impairment of working memory of rats after exposure to sevoflurane was observed. A total of 119 of 7319 detected mRNAs showed significantly different expression between rats with and without sevoflurane exposure (fold change $(F C)>2.0, P<0.05$, and false discovery rate $(F D R)<0.05)$, among which 74 mRNAs were down-regulated and 45 mRNAs were up-regulated. Postsynaptic density-95 (PSD95, also named DLG4) showed the most significantly decreased expression in mPFC and further investigation indicated that PSD95 expression level was correlated with spatial working memory performance. Conclusions: Our study revealed that PSD95 might be involved in the mechanism of POCD, which could provide clues for preventing POCD in clinical operations.
\end{abstract}

Key words: Sevoflurane, Cognitive dysfunction, PSD95 doi: $10.1631 /$ jzus.B1500006

Document code: A
CLC number: R614

\section{Introduction}

Though anesthesia greatly eases the pain of patients undergoing surgical operations, recent studies

\footnotetext{
ॠ Corresponding author

${ }^{\S}$ The two authors contributed equally to this work

* Project supported by the Anhui Education Department (No. KJ2015 B004by) and the Bengbu Medical College (No. BYKY1424ZD), China

\# Electronic supplementary materials: The online version of this article (http://dx.doi.org/10.1631/jzus.B1500006) contains supplementary materials, which are available to authorized users

(D) ORCID: Ye ZHANG, http://orcid.org/0000-0001-6328-8003

(C) Zhejiang University and Springer-Verlag Berlin Heidelberg 2015
}

have shown that exposure to inhaled anesthetics, including isoflurane (Liang et al., 2010) and sevoflurane (Xie et al., 2008), could induce neuropathologic changes, such as neuron apoptosis and increased $\beta$ amyloid protein levels. Besides, evidence from animal models suggested that general anesthesia with volatile anesthetics alone produced abnormal social behaviors resembling autism spectrum disorder (Satomoto et al., 2009) and postoperative cognitive dysfunction (POCD) (Xie et al., 2008; Dong et al., 2009).

Until now, many dysregulated proteins and biological processes, which might be associated with 
POCD caused by sevoflurane exposure, have been identified. For example, Chen et al. (2013) demonstrated that sevoflurane could induce endoplasmic reticulum stress in hippocampal neurons of aged rats. Xiong et al. (2013) also reported that the cyclic adenosine monophosphate (cAMP)/cAMP response element-binding protein (CREB) signaling pathway was down-regulated in aged rats after exposure to sevoflurane. However, the mechanisms underlying POCD caused by sevoflurane still remain largely unknown.

The medial prefrontal cortex (mPFC) is anatomically and functionally linked with subcortical structures including the ventral tegmental area, striatum including nucleus accumbens, and other parts of the mesocorticolimbic circuit (Seamans et al., 2008; Chudasama, 2011). Many studies have demonstrated that $\mathrm{mPFC}$ is implicated in various cognitive impairments, including working memory, attentional processes, decision-making, goal-directed behavior, and behavior flexibility (Goldman-Rakic, 1995; Davidson, 2002; Clark et al., 2004). In the present study, we carried out behavioral tests on Wistar rats exposed to sevoflurane for different time, and observed significant impairment of working memory. We further hypothesized that cognitive impairment caused by sevoflurane exposure was partly due to mPFC dysfunction. To test our hypothesis, we performed mRNA profiling of mPFC from Wistar rats after exposure to sevoflurane and found that dozens of genes were dysregulated, among which was postsynaptic density-95 (PSD95) that was associated with cognitive impairment reported by previous studies (Sultana et al., 2010; Whitfield et al., 2014).

\section{Materials and methods}

\subsection{Animals}

Wistar rats (12-week old, male, and 300-400 g body weight) were purchased from the Beijing Vital River Laboratory Animal Limited Company (Beijing, China). Rats were housed in group cages under a $12-\mathrm{h}$ alternating light/dark cycle with food and water ad libitum in the institutional animal facilities. All housing conditions and experiments were approved and supervised by the Institutional Animal Care and Use Committee of the First Affiliated Hospital of
Bengbu Medical College (Anhui, China). All efforts were made to minimize animal suffering and to reduce the number of animals used.

\subsection{Anesthetic methods}

For behavioral tests, 40 rats were randomly divided into four groups: $0,2,4$, and $6 \mathrm{~h}$ groups. In four groups, rats were exposed to $3 \%$ sevoflurane for 0,2 , 4, and 6 h, respectively (Fig. 1). For anesthesia, rats were placed in a plexiglas anesthesia chamber and exposed to $3 \%$ sevoflurane in a gas mixture of $5 \%$ carbon dioxide $/ 21 \%$ oxygen/balanced nitrogen at the speed of $1.5 \mathrm{~L} / \mathrm{min}$ (Zhou et al., 2013). Sevoflurane, carbon dioxide, and oxygen concentrations of the gas mixture were monitored during the anesthesia.

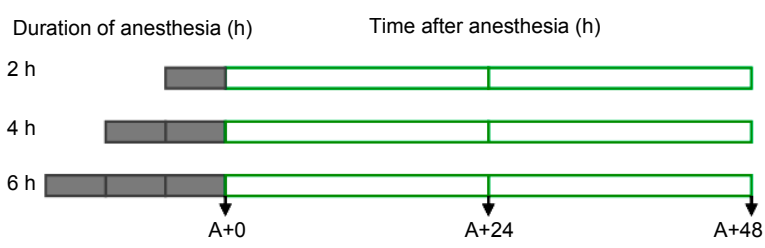

Fig. 1 Timeline of the experiment

Rats were exposed to $3 \%$ sevoflurane for 2,4 , or $6 \mathrm{~h}$. At two time points, namely 24 and $48 \mathrm{~h}$ after anesthesia termination $(\mathrm{A}+24$ and $\mathrm{A}+48)$, rats were subjected to behavioral tests

For gene expression analysis, 100 rats were exposed to $3 \%$ sevoflurane for $2 \mathrm{~h}$ in the above mentioned anesthetizing chamber with the same condition. At the end of anesthesia, animals exposed to sevoflurane were randomly divided into nine groups: $0,3,6$, $12,24,36,48,72$, and $96 \mathrm{~h}$, which represented the time interval from the end of anesthesia to decapitation for gene expression analysis. Another 10 rats were randomly chosen for untreated controls.

During anesthetic exposure, all anesthetized rats were breathing spontaneously, and animal temperature was kept at $(37 \pm 0.5){ }^{\circ} \mathrm{C}$ with a heating pad. Anesthesia was terminated by discontinuing the anesthetics. Rats were allowed to recover for $24 \mathrm{~h}$ to avoid the confounding influence of residual anesthetic.

\subsection{Behavioral experiments}

Rats were tested in a behavioral test battery including the following tasks (in order of testing): elevated plus-maze (EPM), O-maze, and Y-maze. During each test, behaviors were recorded by a video 
camera and then analyzed by an experimenter who was blind to the condition of the animals. Since rats are nocturnal animals, all behavioral tests were conducted during the dark (active) phase between 1:00 a.m. and 4:00 p.m. Before test, rats were transferred, in their home cage, from their housing room to the testing room, where they were kept for $60 \mathrm{~min}$ to familiarize with the testing room.

\subsection{EPM test}

EPM is one of the most widely used assessments to evaluate anxiety-like behavior in rodents ( $\mathrm{Li}$ et al., 2006; Zhang et al., 2014). The EPM apparatus consisted of four arms: two open arms and two closed arms with the same width $\times$ length size $(12 \mathrm{~cm} \times 60 \mathrm{~cm})$ Each arm was attached to a sturdy leg and elevated $60 \mathrm{~cm}$ from the ground. The rat was placed in the center of the maze facing an enclosed arm. Activity in the EPM was measured over a 5-min period as previously described (Zhang et al., 2014). The time spent in and the number of entries into both the open arms and the closed arms were measured using the Noldus tracking system.

\subsection{O-maze test}

The O-maze was a modified apparatus of classic EPM and also applied to evaluate anxiety and exploration. The maze consists of a gray plastic annular runway (width $11 \mathrm{~cm}$, outer diameter $93.5 \mathrm{~cm}$, inner diameter $72 \mathrm{~cm}$ ). The two closed or open runways without a center position prevent any ambiguity in interpretation of the time spent in the different fields. Two opposing sectors were protected by inner and outer walls of gray polyvinyl (height $20 \mathrm{~cm}$ ). Rats were placed in the center of one open runway and allowed to explore for $5 \mathrm{~min}$. The locomotor activities were recorded by View II software automatically. For statistical analysis, the latency for the first exit and total time spent in the open compartments were measured.

\subsection{Y-maze test}

Immediate spatial working memory performance was investigated by recording spontaneous alternation behavior in a Y-maze apparatus consisting of three black-colored acryl arms $(60 \mathrm{~cm} \times 10 \mathrm{~cm} \times$ $45 \mathrm{~cm}$, length $\times$ width $\times$ height), which were positioned at equal angles as described before (Heo et al., 2009). Before test, rats were habituated in the Y-maze re- cording room for $30 \mathrm{~min}$. The rats were placed at the end of one arm and allowed to freely explore the maze for $10 \mathrm{~min}$. Consecutive entry into three arms in an alternative order was defined as a successive entry on overlapping triplet sets (defined as the total number of arm entries minus two, multiplied by 100). Alternation percentage was calculated as the ratio of observed to possible alternations.

\subsection{Expression microarray analysis}

mPFC tissues from rats were homogenized in TRIZOL reagent (Invitrogen, USA) using a Qiagen Tissuelyser (Qiagen, Germany). Total RNA was extracted in accordance with the manufacturer's protocol and then quantified using a NanoDrop ND-1000 spectrophotometer (Thermo Fisher Scientific, Waltham, USA). Integrity of total RNA was assessed using formaldehyde-agarose gel electrophoresis.

Whole genome expression profiling was performed using a GeneChip ${ }^{\circledR}$ Mouse Genome 4302.0 Array (Affymetrix, USA). Normally, $1 \mu \mathrm{g}$ of total RNA was subjected to ribosomal RNA removal using RiboMinus Human/Mouse Transcriptome Isolation Kit (Invitrogen, USA); complementary DNA (cDNA) was then synthesized using Whole-Transcript Sense Target Labeling Assay (Affymetrix, USA), according to the manufacturer's protocols. After fragmentation, biotin-labeled cDNAs were hybridized to arrays at $45^{\circ} \mathrm{C}$ for $17 \mathrm{~h}$, as described in the Affymetrix Users Manual, and then washed and stained using GeneChip ${ }^{\circledR}$ Fluidics Station 450 (Affymetrix, USA).

Upon collection of signal, technical quality control was performed using dChip Version 2005 (Affymetrix, USA) (Li and Wong, 2001) with the default setting. Principal component analysis was performed with Partek Genomics Solution Version 6.5 (Partek, USA). Differential expression analysis between samples was performed using Limma with the functions $1 m$ Fit and eBayes (Smyth, 2004). Gene lists of differentially expressed genes were controlled for false discovery rate (FDR) errors using the method of QVALUE. For fold changes (FCs) of signal between any two conditions, only probesets with Q-values lower than 0.1 and $P$-values lower than 0.05 were retained.

\subsection{Real-time polymerase chain reaction (PCR)}

Single-strand cDNA was synthesized from total RNA by AMV Reverse Transcriptase Kit (Promega, 
Madison, USA) following the standard manufacturer's instructions.

Real-time PCR was performed using LightCycler 480 SYBR Green I Master (Roche, Basel, Switzerland) on a CFX96 Real-Time PCR Detection System (Bio-Rad, USA). The PCR conditions included an initial step at $95^{\circ} \mathrm{C}$ for $5 \mathrm{~min}$, followed by 40 cycles of amplification and quantification $\left(95^{\circ} \mathrm{C}\right.$ for $10 \mathrm{~s}, 60{ }^{\circ} \mathrm{C}$ for $20 \mathrm{~s}$, and $72{ }^{\circ} \mathrm{C}$ for $30 \mathrm{~s}$ ). Each cDNA sample was performed in triplicates in a final volume of $20 \mu \mathrm{l}$ containing $1 \mu \mathrm{l}$ of cDNA and $400 \mathrm{nmol} / \mathrm{L}$ of forward and reverse gene-specific primers. Relative gene expression levels were quantified based on the cycle threshold values and normalized to the reference gene actin. All specific primers were available upon request.

\subsection{Statistical analysis}

All statistical analyses were done using SPSS 17.0 software and GraphPad Prism 5. For behavioral tests, repeated measurement analysis of variance (ANOVA) was applied for comparison among groups with Bonferroni post-tests. For real-time PCR, the statistical significance of the difference among groups was evaluated by one-way ANOVA with Tukey's post hoc multiple comparisons. Correlation analysis was performed using Pearson's correlation coefficients. All data were represented as mean \pm standard error of the mean (SEM), and a $P$-value of $<0.05$ was considered statistically significant.

\section{Results}

\subsection{Sevoflurane caused spatial working memory impairment in aged rats}

There was no significant difference of exploration in the open arms in the EPM test either 24 or $48 \mathrm{~h}$ after the termination of anesthesia between rats with and without $3 \%$ sevoflurane exposure (two-way ANOVA, $P>0.05$ for each comparison with $0 \mathrm{~h}$ group; Fig. 2a). Total activity, manifested as similar total number of arm entries, also showed no significant difference (two-way ANOVA, $P>0.05$ for each comparison with 0 h group; Fig. 2b).

As shown in Figs. 2c and 2d, both the latency to enter the anxiety-related bright compartment and the time spent on the aversive open sector in the O-maze test were consistent between rats with and without sevoflurane exposure for both test time points (two-way ANOVA, $P>0.05$ for each comparison with $0 \mathrm{~h}$ group).

In the Y-maze task, rats faced a choice in selecting a pathway in the Y-shaped track. Spontaneous alterations in arm entries were significantly lower in rats exposed to $3 \%$ sevoflurane compared with the

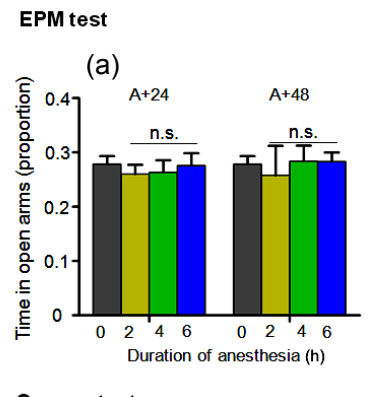

(b)

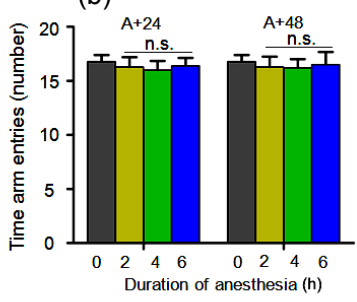

(c)

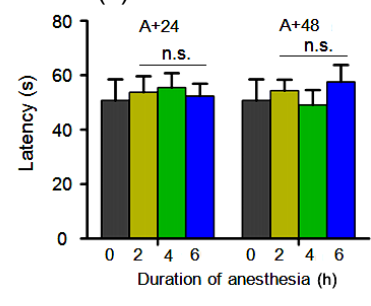

(d)

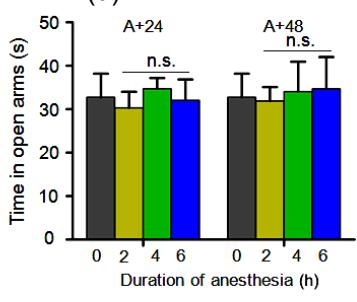

(f)
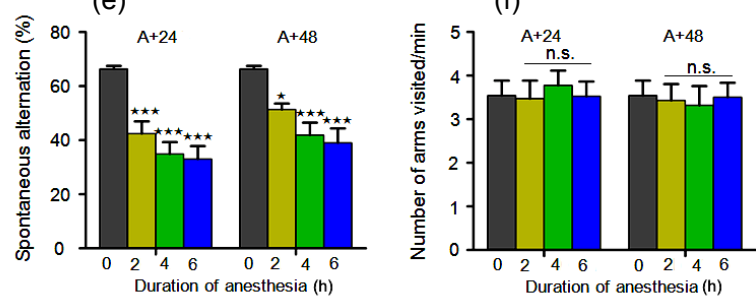

Fig. 2 Behavioral tests of rats exposed to $3 \%$ sevoflurane for $0,2,4$, and $6 \mathrm{~h}$, respectively

In EPM test, there was no significant difference in time spent in open arms (a) and total arm entries (b) between normal rats and those exposed to 3\% sevoflurane for 2-6 h. In O-maze test, latencies to venture out on the open arm (c) as well as time spent in open arms (d) were similar between rats with and without 3\% sevoflurane exposure. In Y-maze test, exposure to $3 \%$ sevoflurane did not influence the number of arms visited per min (f) but led to decreased spontaneous alternations (e). $\mathrm{A}+24$ and $\mathrm{A}+48$ represented two time points, namely 24 and $48 \mathrm{~h}$ after the end of anesthesia. Data are expressed as mean \pm SEM ( $n=8-10$ rats per group). The data were analyzed using repeated measurement ANOVA. ${ }^{\star} P<0.05,{ }^{\star \star} P<0.01$, and ${ }^{\star \star \star} P<0.001$, which were determined using Bonferroni's post hoc test, represent significant differences versus the $0 \mathrm{~h}$ group. n.s.: not significant 
untreated rats (two-way ANOVA, $P<0.05$ for each comparison with $0 \mathrm{~h}$ group; Fig. 2e). It is worth noting that performance in spontaneous alterations improved slightly in rats tests $48 \mathrm{~h}$ after anesthesia termination, compared with rats tested $24 \mathrm{~h}$ after anesthesia termination, for each anesthesia time, respectively (Fig. 2e). The number of arm entries was not substantially different across all groups (Fig. 2f), indicating that alterations in behavior are not caused by changed movements.

\subsection{Sevoflurane induced mRNA profiling dysregulation in mPFC of aged rats}

From Fig. 2e, we knew that exposure to $3 \%$ sevoflurane for $2 \mathrm{~h}$ was enough to cause spatial working memory impairment in aged rats. In order to investigate the genes responsible for such impairment, we performed mRNA profiling of mPFC of rats $(n=3)$ $24 \mathrm{~h}$ after termination of $3 \%$ sevoflurane exposure $(2 \mathrm{~h})$, at which time spatial working memory of rats was noticeably impaired. Another 3 rats without 3\% sevoflurane exposure were used as background controls.

From the analysis, we detected a total of 7319 mRNAs, of which 119 showed a significantly different expression between experimental rats and controls (FC $>2.0, P<0.05$, and FDR $<0.05$; Supplementary Table S1). Among them, 74 mRNAs were down-regulated and 45 were up-regulated. Their distinct expression patterns were presented by hierarchical clustering analysis (Fig. 3a). We randomly selected 10 dysregulated mRNAs, including 5 down-regulated (DLG4, IL1RL1, CCL8, GLDN, and GRIDI) and 5 up-regulated (RBAK, CFH, CREBRF, CEP350, and $R A S E F$ ), for verification in these $\mathrm{mPFC}$ tissues. A general consistency between the real-time PCR and microarray analysis results was confirmed in 9 selected mRNAs in terms of regulation direction (up-regulation or down-regulation) and significance except $R B A K$ (Fig. 3b).

\subsection{PSD95 expression is correlated with spatial working memory performance of rats after ex- posure to sevoflurane}

Since PSD95 (also named DLG4) showed dramatically decreased expression in $\mathrm{mPFC}$ from microarray data, we further investigated whether PSD95 expression level was correlated with spatial working memory performance. A total of 90 rats were randomly divided into 9 groups (10 rats in each group) and exposed to $3 \%$ sevoflurane for $2 \mathrm{~h}$. We analyzed PSD95 expression at 9 time points corresponding to the 9 groups, namely $0,3,6,12,24,36,48,72$, and $96 \mathrm{~h}$ after termination of the $3 \%$ sevoflurane exposure. Since rats were allowed to recover for $24 \mathrm{~h}$ to avoid the confounding influence of residual anesthetic, we also performed the Y-maze test at 5 time points, namely 24, 36, 48, 72, and $96 \mathrm{~h}$ after anesthesia.

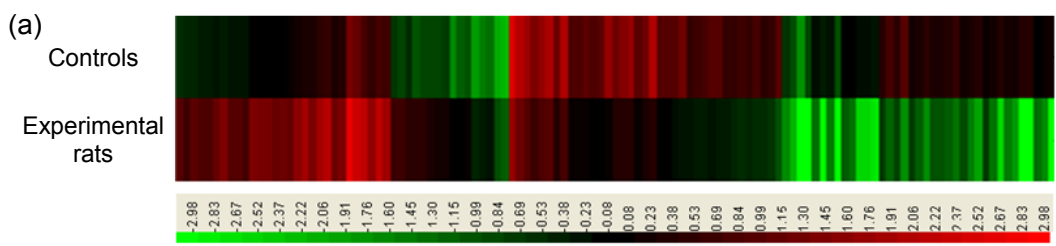

(b)

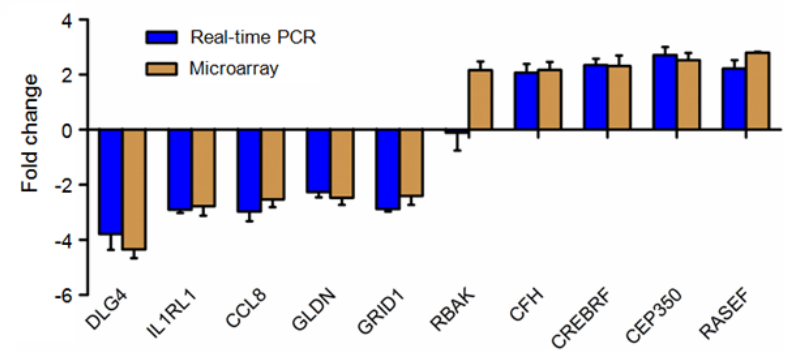

Fig. 3 Dysregulated genes in the mPFC of rats after exposure to $3 \%$ sevoflurane for $3 \mathrm{~h}$

(a) The hierarchical clustering of partial differentially expressed mRNAs. "Red" indicates high relative expression, and "green" indicates low relative expression. (b) Real-time PCR validation of 10 differentially expressed mRNAs from microarray data. Fold changes were calculated by $2^{-\Delta C_{\mathrm{T}}}$, where $\Delta C_{\mathrm{T}}=C_{\mathrm{T}}$, target $-C_{\mathrm{T}}$, actin. Data are expressed as mean $\pm \operatorname{SEM}(n=3)$ (Note: for interpretation of the references to color in this figure legend, the reader is referred to the web version of this article) 
Interestingly, the expression of PSD95 started to decrease at $6 \mathrm{~h}(\mathrm{FC}=0.76, P>0.05)$ after the termination of anesthesia, reached the lowest level at $36 \mathrm{~h}$ ( $\mathrm{FC}=0.26, P<0.001)$, and then gradually recovered to normal levels at the 96-h time point (Fig. 4a). For the performance of spatial working memory in experimental rats, we observed the most severe impairment at the 36-h time point (Fig. 4b). Further analysis showed that the expression of PSD95 was positively correlated with spatial working memory in experimental rats $\left(r^{2}=0.6529, P=0.046\right.$; Fig. $\left.4 \mathrm{c}\right)$. We also observed that spatial working memory of experimental rats did not recover to normal levels as controls at the 96-h time point, suggesting that, apart from PSD95, other genes might be involved in the regulation of spatial working memory.

\section{Discussion}

In the present study, we found that exposure to $3 \%$ sevoflurane for $2 \mathrm{~h}$ led to spatial impaired working memory, one of the characteristics of cognitive function, in rats. Cognitive function is one of the most basic and important high-level neurological functions in the brain, and it is a basic indicator of the development of human intelligence. Supporting our result, many previous studies also reported the effect of sevoflurane, as well as other anesthetics, on cognitive function (Chen et al., 2001; Millar et al., 2006; Peng et al., 2011; Gong et al., 2012). Interestingly, Peng et al. (2011) found that exposure to $1.5 \%$ sevoflurane, for as long as $3 \mathrm{~d}(2 \mathrm{~h} / \mathrm{d})$, did not cause significantly different cognitive performance, while exposure to $3 \%$ sevoflurane for $2 \mathrm{~h}$ was enough to induce cognitive impairment. This evidence suggests that the concentration of sevoflurane used to induce or to sustain anesthesia should be as low as possible.

It is a quite complex question as to why cognitive function is impaired after exposure to sevoflurane, and the underlying mechanisms remain largely unknown. Ji et al. (2015) reported that sevoflurane exposure could induce cognitive impairment later in life partly through neural apoptosis, inflammation, and oxidative nitrosative stress in mouse brain. Hu et al. (2014) revealed that opening of the blood-brain barrier was related to cognitive decline in aged rats following high concentration of sevoflurane inhalation. (a)

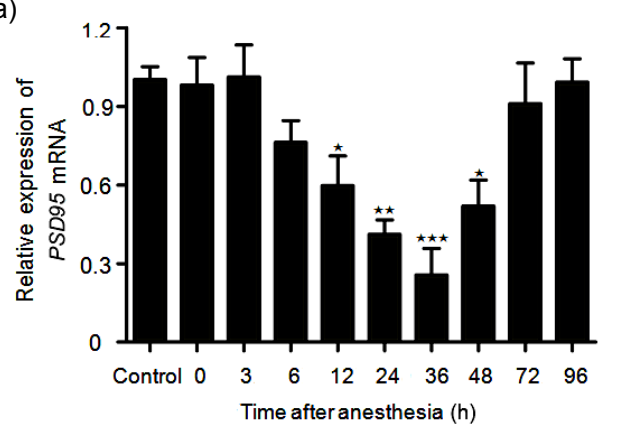

(b)

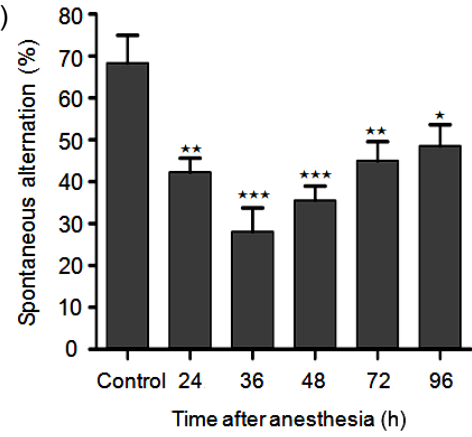

(c)

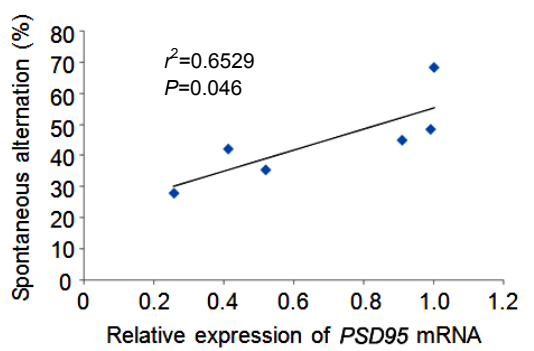

Fig. 4 Expression of $P S D 95$ in mPFC and performance of spatial working memory of rats after exposure to sevoflurane (a) Expression of PSD95 in mPFC was analyzed by real-time PCR at 9 time points after exposure to sevoflurane. (b) Analysis of spontaneous alternations in Y-maze test at 5 time points after the termination of 3\% sevoflurane exposure. 0-96 h represent the time intervals from termination of anesthesia to analysis. Data shown were representative of six independent experiments and error bars represented mean $\pm \operatorname{SEM}(n=10)$. The data were analyzed using one-way ANOVA. ${ }^{\star} P<0.05,{ }^{\star}{ }^{\star} P<0.01$, and ${ }^{\star \star \star} P<0.001$, compared with the control. (c) PSD95 expression was positively correlated with spatial working memory in experimental rats $\left(r^{2}=0.6529, P=0.046\right)$ 
In our study, we found that PSD95 expression in $\mathrm{mPFC}$ was down-regulated $6 \mathrm{~h}$ after termination of sevoflurane exposure and then up-regulated to normal levels at the 96-h time point. To our knowledge, this is the first time that decreased PSD95 in $\mathrm{mPFC}$ has been implicated in the mechanisms of sevofluraneinduced cognitive impairment. PSD95 is predominantly expressed in the brain (hippocampus CA1 region as well as prefrontal cortex), where it localizes in a somatodendritic pattern in the post-synaptic membrane and in presynaptic axon terminals of inhibitory neurons (Kim et al., 1995). Many studies have investigated the roles of PSD95. For example, it was reported that neuronal nitric-oxide synthase (nNOS)PSD95 protein-protein interaction is important in maintaining hypersensitivity in acute and chronic pain and disruption of the nNOS-PSD95 interaction could inhibit acute thermal hyperalgesia and chronic mechanical allodynia (Florio et al., 2009). PSD95 was also implicated in the regulation of ischemic injury through nNOS-PSD95 (Hu et al., 2013) interaction and the glutamate receptor 6 (GluR6)-PSD95calmodulin-dependent protein kinase II (CaMKII) signaling module (Xu et al., 2010). However, whether and how decreased PSD95 expression was involved in cognitive impairment remains elusive. Several studies have implied that decreased PSD95 could lead to disrupted synaptic structures (Rubino et al., 2009) and neuron apoptosis (Sultana et al., 2010). We believe these observations could provide clues to investigate the functions of PSD95 in cognitive function.

Another interesting phenomenon is that even though the expression of PSD95 recovered to normal levels, the spatial working memory of rats was still worse than that of normal rats. We think that this might be partly due to residual sevoflurane in the nervous system. Another more reasonable explanation is that, apart from PSD95, many other proteins are involved in the regulation of cognitive function. Possible proteins include GRB10 (Xie et al., 2014), NGF (Wang et al., 2014), ZNF407 (Kambouris et al., 2014). We also could not exclude the possibility that non-coding RNAs might be involved in the pathogenesis of cognitive impairment, since previous studies have shown various regulatory roles (Rinn and Chang, 2012). It would be meaningful to systematically investigate the mechanisms underlying the cognitive impairment induced by sevoflurane exposure.

\section{Acknowledgements}

We would like to thank all of the rats for their lives. We thank Dr. Shu-quan RAO (Chengdu University of Traditional Chinese Medicine) for helpful discussion, and Yang-kui ZHAI (Tianjin Medical University Metabolic Diseases Hospital) for technical assistance.

\section{Compliance with ethics guidelines}

Yun-zhi LING, Wei MA, Li YU, Ye ZHANG, and Qi-sheng LIANG declare that they have no conflict of interest. All institutional and national guidelines for the care and use of laboratory animals were followed.

\section{References}

Chen, G., Gong, M., Yan, M., et al., 2013. Sevoflurane induces endoplasmic reticulum stress mediated apoptosis in hippocampal neurons of aging rats. PLOS ONE, 8(2): e57870. [doi:10.1371/journal.pone.0057870]

Chen, X., Zhao, M., White, P.F., et al., 2001. The recovery of cognitive function after general anesthesia in elderly patients: a comparison of desflurane and sevoflurane. Anesth. Analg., 93(6):1489-1494. [doi:10.1097/00000539200112000-00029]

Chudasama, Y., 2011. Animal models of prefrontal-executive function. Behav. Neurosci., 125(3):327-343. [doi:10. 1037/a0023766]

Clark, L., Cools, R., Robbins, T.W., 2004. The neuropsychology of ventral prefrontal cortex: decision-making and reversal learning. Brain Cogn., 55(1):41-53. [doi:10. 1016/S0278-2626(03)00284-7]

Davidson, R.J., 2002. Anxiety and affective style: role of prefrontal cortex and amygdala. Biol. Psychiatry, 51(1): 68-80. [doi:10.1016/S0006-3223(01)01328-2]

Dong, Y.L., Zhang, G.H., Zhang, B., et al., 2009. The common inhalational anesthetic sevoflurane induces apoptosis and increases $\beta$-amyloid protein levels. Arch. Neurol., 66(5): 620-631. [doi:10.1001/archneurol.2009.48]

Florio, S.K., Loh, C., Huang, S.M., et al., 2009. Disruption of nNOS-PSD95 protein-protein interaction inhibits acute thermal hyperalgesia and chronic mechanical allodynia in rodents. Br. J. Pharmacol., 158(2):494-506. [doi:10.1111/ j.1476-5381.2009.00300.x]

Goldman-Rakic, P.S., 1995. Architecture of the prefrontal cortex and the central executive. Ann. N. Y. Acad. Sci., 769:71-83. [doi:10.1111/j.1749-6632.1995.tb38132.x]

Gong, M., Chen, G., Zhang, X.M., et al., 2012. Parecoxib mitigates spatial memory impairment induced by sevoflurane anesthesia in aged rats. Acta Anaesthesiol. Scand., 56(5):601-607. [doi:10.1111/j.1399-6576.2012. 02665.x]

Heo, H., Shin, Y., Cho, W., et al., 2009. Memory improvement 
in ibotenic acid induced model rats by extracts of Scutellaria baicalensis. J. Ethnopharmacol., 122(1): 20-27. [doi:10.1016/j.jep.2008.11.026]

$\mathrm{Hu}, \mathrm{N}$., Guo, D., Wang, H., et al., 2014. Involvement of the blood-brain barrier opening in cognitive decline in aged rats following orthopedic surgery and high concentration of sevoflurane inhalation. Brain Res., 1551:13-24. [doi:10. 1016/j.brainres.2014.01.015]

Hu, Z., Bian, X., Liu, X., et al., 2013. Honokiol protects brain against ischemia-reperfusion injury in rats through disrupting PSD95-nNOS interaction. Brain Res., 1491: 204-212. [doi:10.1016/j.brainres.2012.11.004]

Ji, M.H., Qiu, L.L., Yang, J.J., et al., 2015. Pre-administration of curcumin prevents neonatal sevoflurane exposureinduced neurobehavioral abnormalities in mice. Neurotoxicology, 46:155-164. [doi:10.1016/j.neuro.2014 11.003]

Kambouris, M., Maroun, R.C., Ben-Omran, T., et al., 2014. Mutations in zinc finger 407 [ZNF407] cause a unique autosomal recessive cognitive impairment syndrome. Orphanet J. Rare Dis., 9(1):80. [doi:10.1186/1750-11729-80]

Kim, E., Niethammer, M., Rothschild, A., et al., 1995. Clustering of Shaker-type $\mathrm{K}^{+}$channels by interaction with a family of membrane-associated guanylate kinases. Nature, 378(6552):85-88. [doi:10.1038/378085a0]

Li, C., Wong, W.H., 2001. Model-based analysis of oligonucleotide arrays: expression index computation and outlier detection. PNAS, 98(1):31-36. [doi:10.1073/pnas. 98.1.31]

Li, S., Murakami, Y., Wang, M., et al., 2006. The effects of chronic valproate and diazepam in a mouse model of posttraumatic stress disorder. Pharmacol. Biochem. Behav., 85(2):324-331. [doi:10.1016/j.pbb.2006.08.015]

Liang, G., Ward, C., Peng, J., et al., 2010. Isoflurane causes greater neurodegeneration than an equivalent exposure of sevoflurane in the developing brain of neonatal mice. Anesthesiology, 112(6):1325-1334. [doi:10.1097/ALN. 0b013e3181d94da5]

Millar, K., Asbury, A.J., Bowman, A.W., et al., 2006. The effects of brief sevoflurane-nitrous oxide anaesthesia upon children's postoperative cognition and behaviour. Anaesthesia, 61(6):541-547. [doi:10.1111/j.1365-2044. 2006.04662.x]

Peng, S., Zhang, Y., Sun, D.P., et al., 2011. The effect of sevoflurane anesthesia on cognitive function and the expression of insulin-like growth factor-1 in CA1 region of hippocampus in old rats. Mol. Biol. Rep., 38(2): 1195-1199. [doi:10.1007/s11033-010-0217-9]

Rinn, J.L., Chang, H.Y., 2012. Genome regulation by long noncoding RNAs. Annu. Rev. Biochem., 81(1):145-166. [doi:10.1146/annurev-biochem-051410-092902]

Rubino, T., Realini, N., Braida, D., et al., 2009. Changes in hippocampal morphology and neuroplasticity induced by adolescent THC treatment are associated with cognitive impairment in adulthood. Hippocampus, 19(8):763-772. [doi:10.1002/hipo.20554]

Satomoto, M., Satoh, Y., Terui, K., et al., 2009. Neonatal exposure to sevoflurane induces abnormal social behaviors and deficits in fear conditioning in mice. Anesthesiology, 110(3):628-637. [doi:10.1097/ALN.0b0 13e3181974fa2]

Seamans, J.K., Lapish, C.C., Durstewitz, D., 2008. Comparing the prefrontal cortex of rats and primates: insights from electrophysiology. Neurotox. Res., 14(2-3):249-262. [doi:10.1007/BF03033814]

Smyth, G.K., 2004. Linear models and empirical bayes methods for assessing differential expression in microarray experiments. Stat. Appl. Genet. Mol. Biol., 3(1):1-25. [doi:10.2202/1544-6115.1027]

Sultana, R., Banks, W.A., Butterfield, D.A., 2010. Decreased levels of PSD95 and two associated proteins and increased levels of $\mathrm{BCl}_{2}$ and caspase 3 in hippocampus from subjects with amnestic mild cognitive impairment: insights into their potential roles for loss of synapses and memory, accumulation of $A \beta$, and neurodegeneration in a prodromal stage of Alzheimer's disease. J. Neurosci. Res., 88(3):469-477. [doi:10.1002/jnr.22227]

Wang, F., Chang, G., Geng, X., 2014. NGF and TERT co-transfected BMSCs improve the restoration of cognitive impairment in vascular dementia rats. PLoS ONE, 9(6):e98774. [doi:10.1371/journal.pone.0098774]

Whitfield, D.R., Vallortigara, J., Alghamdi, A., et al., 2014. Assessment of ZnT3 and PSD95 protein levels in Lewy body dementias and Alzheimer's disease: association with cognitive impairment. Neurobiol. Aging, 35(12): 2836-2844. [doi:10.1016/j.neurobiolaging.2014.06.015]

Xie, J., Wei, Q., Deng, H., et al., 2014. Negative regulation of GRB10 interacting GYF protein 2 on insulin-like growth factor-1 receptor signaling pathway caused diabetic mice cognitive impairment. PLoS ONE, 9(9):e108559. [doi:10. 1371/journal.pone.0108559]

Xie, Z.C., Culley, D.J., Dong, Y.L., et al., 2008. The common inhalation anesthetic isoflurane induces caspase activation and increases amyloid $\beta$-protein level in vivo. Ann. Neurol., 64(6):618-627. [doi:10.1002/ana.21548]

Xiong, W.X., Zhou, G.X., Wang, B., et al., 2013. Impaired spatial learning and memory after sevoflurane-nitrous oxide anesthesia in aged rats is associated with downregulated cAMP/CREB signaling. PLOS ONE, 8(11): e79408. [doi:10.1371/journal.pone.0079408]

Xu, J., Liu, Z.A., Pei, D.S., et al., 2010. Calcium/calmodulindependent kinase II facilitated GluR6 subunit serine phosphorylation through GluR6-PSD95-CaMKII signaling module assembly in cerebral ischemia injury. Brain Res., 1366:197-203. [doi:10.1016/j.brainres.2010.09.087]

Zhang, W., Hetzel, A., Shah, B., et al., 2014. Greater physiological and behavioral effects of interrupted stress pattern compared to daily restraint stress in rats. PLoS ONE, 9(7):e102247. [doi:10.1371/journal.pone.0102247] 
Zhou, H., Li, S., Niu, X., et al., 2013. Protective effect of FTY720 against sevoflurane-induced developmental neurotoxicity in rats. Cell Biochem. Biophys., 67(2): 591-598. [doi:10.1007/s12013-013-9546-3]

\section{List of electronic supplementary materials}

Table S1 Dysregulated mRNAs in in medial prefrontal cortex between experimental rats and normal rats

\section{中文概要}

题 目: 七氟烷麻醉诱导的认知功能损伤与内测前额叶皮 层中 PSD95 表达量降低有关
目 的: 临床上接受七氟烷麻醉的病人, 术后会出现认知 功能损伤。本研究旨在挖掘七氟烷麻醉导致的内 侧前额叶皮层中基因表达谱的改变, 并探讨术后 认知功能改变的机制。

创新点: 本研究深入探讨七氟烷麻醉导致认知功能损伤的 分子机制, 充分利用表达谱和动物行为学实验来 揭示可能的分子机制。

方 法: 采用十字迷宫、O 迷宫和水迷宫分析接受不同时 间七氟烷处理的 Wistar 大鼠的行为学特征; 同时 提取实验大鼠内侧前额叶皮层的 mRNA 进行表 达谱分析 (图 3) , 采用实时聚合酶链反应 （real-time PCR）对差异表达 mRNA 进行验证。

结 论: 内侧前额叶皮层中 PSD95 表达量降低与七氟烷 麻醉诱导的认知功能损伤有关。

关键词: 七氟烷; 认知功能损伤; PSD 95 